\title{
RADIO AND X-RAY EMISSIONS FROM CHEMICALLY PECULIAR B- AND A-TYPE STARS: OBSERVATIONS AND A MODEL
}

\author{
JEFFREY L. LINSKY ${ }^{1}$ \\ Joint Institute for Laboratory Astrophysics, National Institute of Stan- \\ dards and Technology and University of Colorado, Boulder, CO 80309- \\ 0440 .
}

\begin{abstract}
Conventional wisdom holds that early-type and late-type stars have very different outer atmospheres, because the early-type stars lack deep convective zones. I argue that the magnetic chemically peculiar (CP) stars hotter than about spectral type A2 display many of the activity phenomena seen in the most active late-type stars. In particular, many $\mathrm{CP}$ stars are luminous nonthermal radio and coronal $\mathrm{x}$-ray sources like the RS $\mathrm{CV}_{n}$ systems. A wind-fed magnetosphere model has been proposed to explain both the nonthermal radio and the $\mathrm{x}$-ray emission. In this model the stellar wind plays the role of a mechanical energy source analogous to the role played by convection in the active late-type stars.
\end{abstract}

\section{IS CONVENTIONAL WISDOM CORRECT FOR CP STARS?}

Since the first convincing discovery of a flare on a star (UV Ceti) other than the Sun by Luyten (1949), astronomers have studied solar-like phenomena on latetype stars in increasing detail. Included among these phenomena are chromospheres, multimillion degree coronae, nonthermal radio emission, dark starspots, active regions, flares, and other transient or nonclassical phenomena observed across the electromagnetic spectrum. The apparent absence of these phenomena in the A- and B-type stars has been the basis for a widely held belief that the outer atmospheres of early-type and late-type stars are fundamentally different: early-type stars lack the deep convective zones that presumably are required for the dynamo generation of turbulent magnetic fields, nonradiative heating of the outer layers, and the acceleration of nonthermal electrons.

I have long felt that this "conventional wisdom" was at minimum too simplistic and perhaps completely wrong. My opinion was based on the presence of strong magnetic fields in the photospheres of many slowly rotating chemically peculiar (CP) A- and B-type stars measured by Babcock (1958) and subse-

\footnotetext{
${ }^{1}$ Staff member, Quantum Physics Division, National Institute of Standards and Technology
} 
quent workers. These fields are easily measured because they are ordered (simple dipoles or quadrupoles), unlike the complex, turbulent fields observed on the Sun and presumed to be present on active late-type stars. If such large fields (up to $34 \mathrm{kG}$ for the mean surface field in the case of Babcock's star) are observed on some stars, might not magnetic fields also be present but not detected on other A- and B-type stars? This could occur because conventional magnetometers that measure Zeeman lines in polarized light have great difficulty measuring fields with a complex geometry like the Sun due to field cancellation or in the presence of rapid stellar rotation.

Strong magnetic fields are necessary but not sufficient conditions for solarlike activity. All that would be required for active phenomena on the magnetic $A$ and B-type stars is a source of mechanical energy, like convection in the late-type stars. The presence of chemical peculiarities is generally explained by diffusion, which does not operate where convection (and presumably other sources of mechanical energy) are found. This logic serves as a theoretical explanation for the apparent absence of "active" phenomena in these stars. Table I summarizes the conventional wisdom concerning magnetic CP stars prior to 1985 . X-ray and 6-cm radio luminosities are given for the Sun and the RS CVn systems, which are among the most active of the late-type stars as indicated by their large $\mathrm{x}$ ray and radio luminosities and their energetic flares. The more active star in a RS CVn system is typically an early $\mathrm{K} 0$ subgiant with a deep convective zone and large rotational velocity due to tidally-induced synchronism of rotation and revolution. For more information see, for example, Strassmeier et al. (1988).

TABLE I. Summary of Conventional Wisdom

\begin{tabular}{|c|c|c|}
\hline Stellar Property & CP Stars & Active Cool Stars \\
\hline 1. Convective zone & No & Yes \\
\hline 2. Stable atmosphere & Yes & No \\
\hline 3. Magnetic field & Ordered & Complex, flux tubes, plages \\
\hline 4. Rotation rate & Slow for spectral type & Fast for spectral type \\
\hline 5. Winds & $\begin{array}{c}\text { Indirect evidence } \\
\text { (polar jets) }\end{array}$ & Yes in open field regions \\
\hline $\begin{array}{l}\text { 6. Hot coronae } \\
\text { (x-ray emission) }\end{array}$ & $\begin{array}{l}\text { No conclusive } \\
\text { evidence }\end{array}$ & $\begin{array}{c}\log \mathrm{L}_{x}^{\odot}=27.0-28.0 \\
\log \mathrm{L}_{x}^{R S}=29.5-32.0\end{array}$ \\
\hline $\begin{array}{l}\text { 7. Nonthermal radio } \\
\text { emission }\end{array}$ & $\begin{array}{c}\text { No evidence before } \\
1985\end{array}$ & $\begin{array}{c}\log \mathrm{L}_{6}^{\odot}=10.5-12.0 \\
\log L_{6}^{R S}=14.5-18.0\end{array}$ \\
\hline $\begin{array}{l}\text { 8. Coronal heating } \\
\text { mechanism }\end{array}$ & Not required & $\begin{array}{c}\text { MHD Processes } \\
\text { (recombination or shocks) }\end{array}$ \\
\hline $\begin{array}{l}\text { 9. Electron accel. } \\
\text { mechanism }\end{array}$ & Not required & $\begin{array}{l}\text { Magnetic field reconnection } \\
\text { produces electric fields }\end{array}$ \\
\hline
\end{tabular}

In this talk I will ask the question: Are the magnetic chemically peculiar stars high-temperature analogs of the active cool stars? To answer this heretical question, I will summarize what we have been learning about the nonthermal radio and high-temperature $\mathrm{x}$-ray emission from these stars. I will 
also describe a magnetosphere model that we have developed for these stars in which weak radiation-driven winds provide the mechanical energy source required with the strong magnetic fields for the active phenomena. Thus the winds in the A- and B-type CP stars play a role analogous to convection in the late-type stars.

\section{FIRST TEST OF CONVENTIONAL WISDOM: THE SEARCH FOR NONTHERMAL RADIO EMISSION}

Stimulated perhaps by the correlation of strong magnetic fields with radio emission on the Sun, several groups have searched unsuccessfully for radio emission from CP stars (see Drake et al. 1987 for a summary). Drake et al. (1985) reported the first radio detections of the CP star $\sigma$ Ori E (B2Vp He-S), discovered serendipitously, and another helium strong (He-S) star HR 1890 (B1.5V He-S), together with 12 nondetections. This minisurvey revealed two properties of these stars that would be confirmed in subsequent surveys: the radio spectra are typically flat between 2 and $6 \mathrm{~cm}$, which is consistent with gyrosynchrotron emission from a nonthermal distribution of electrons, and the high radio luminosity of some sources. For example, $\log L_{6}=17.8$ for $\sigma$ Ori $\mathrm{E}$, which is nearly $10^{7}$ times more luminous than the Sun.

In their 6-cm VLA survey of 34 CP stars, Drake et al. (1987) increased the list of detections from two to five stars. They detected three He-S stars with early-B spectral types ( $\delta$ Ori $\mathrm{C}$ was added to the previous detections HR 1890 and $\sigma$ Ori E), and for the first time detected two helium weak (He-W) and silicon strong stars with late-B or A0 spectral types (IQ Aur and GL Lac). The latter is often called Babcock's star. They noted that no classic Ap stars with enhanced abundances of $\mathrm{Sr}, \mathrm{Cr}$ and Eu were detected, even though the survey sensitivity extended down to values of $\mathrm{L}_{6} 200$ times smaller than the luminosity of $\sigma$ Ori $\mathrm{E}$. The $\mathrm{SrCrEu}$ stars are cooler than the $\mathrm{He}-\mathrm{W}$ stars, which in turn are cooler than the He-S stars. We will show later that this sequence of effective temperatures plays a critical role in explaining the origin of the radio emission. Drake et al. (1987) provided additional support for the idea that gyrosynchrotron emission from mildly relativistic electrons is the emission mechanism by showing that the radio spectra are flat between 2 and $20 \mathrm{~cm}$, the emission from $\sigma$ Ori $\mathrm{E}$ and $\mathrm{HR}$ 1890 is variable on time scales of hours, and the radio luminosities of all detected stars are very large.

Linsky, Drake, and Bastian's (1992) detection of 11 additional He-W stars at $6 \mathrm{~cm}$ allowed them to identify statistical properties of the sample and to develop a model for the magnetospheres of these stars. They noted that on average the $\mathrm{He}-\mathrm{S}$ stars are 20 times more radio luminous than the cooler $\mathrm{He}-\mathrm{W}$ stars ( $\left\langle\log \mathrm{L}_{6}\right\rangle=17.7$ compared with 16.4 for the He-W stars) and 1000 times more luminous than $\theta \mathrm{Aur}(\mathrm{A0pSi})$, which is the coolest and least radio luminous star detected. With 16 detections and 46 upper limits, they were able to find meaningful correlations with effective temperature $\left(\mathrm{L}_{6} \sim T_{\text {eff }}^{6.6}\right)$ and with the root mean square value of the photospheric magnetic field $\left(\mathrm{L}_{6} \sim B_{\mathrm{RMS}}^{1.36}\right)$. They found 
no significant correlation, however, with stellar rotational velocity. In a poster at this meeting, Leone and Umana show that the radio emission from $\sigma$ Ori $\mathrm{E}$ and HD 37017 varies with rotational phase (see also Leone 1991).

Although mass loss rates have not been measured for these stars, radiationdriven wind theory predicts that the mass loss rate $(\dot{M})$ should depend on a high power of $\mathrm{T}_{\text {eff }}$. Linsky et al. conclude that $\mathrm{L}_{6} \sim \dot{\mathrm{M}}^{0.49} B_{\mathrm{RMS}}^{0.95}$, with a correlation coefficient of 0.90 . This indicates that mass loss can play a critical role in the radio emission process. They also conclude that the magnetic $\mathrm{CP}$ stars must be a new class of radio stars as the radio emission from the active cool stars does not depend on the mass loss rate, and the radio emission from the O-type stars depends on the mass loss rate but not, apparently, on the magnetic field strength.

\section{A Wind-fed Magnetosphere Model}

Linsky et al. (1992) proposed that the observed properties of radio emission from the magnetic CP stars may be understood as optically thick gyrosynchrotron emission from a nonthermal distribution of electrons produced in a current sheet far from the star. Their model (see Fig. 1) consists of a rotating dipolar magnetosphere in which stellar wind plasma flows out from near the magnetic poles along the field lines. Far from the star $\left(r \gtrsim 10 R_{\star}\right)$, the gas pressure exceeds the magnetic pressure and the field lines near the equator are drawn out into current sheet configurations (see Havnes and Goertz 1984) analogous to planetary magnetotails. These current sheets are likely locations of plasma heating and particle acceleration, as strong magnetic field gradients produce electric fields and tearing mode instabilities may be important. In the complex geometry provided by the usual displacement of the rotational and magnetic poles, there are many possible scenarios for particle acceleration and heating. In their "direct injection" scenario, the electrons return to near the star by traveling along magnetic fields, where they are reflected and radiate in the strong fields near the magnetic poles.

The model proposed by Linsky et al. accounts for time variability in terms of intrinsic variability in the acceleration process and rotational modulation of the projected area of the emitting region. The flat microwave spectra result from two factors: (i) a decrease in source size with increasing frequency and (ii) a decrease in effective temperature of the emitting electrons with increasing frequency. The finite degree of circular polarization observed on some sources may be the result of an opacity effect resulting from the increasing magnetic field strength with decreasing radius. Finally, the decreasing radio luminosity with decreasing stellar effective temperature along the main sequence is explained primarily as a result of the decreasing mass loss rate for radiation driven winds.

While both the CP stars and the RS CVn systems radiate by the gyrosynchrotron process with similar radio luminosities (see Table II and Fig. 2), the physical processes leading to electron acceleration are likely different. For CP stars, the radio emission requires a wind to distend the field lines, create current sheets, and accelerate the electrons far from the star. In the RS CVn model 


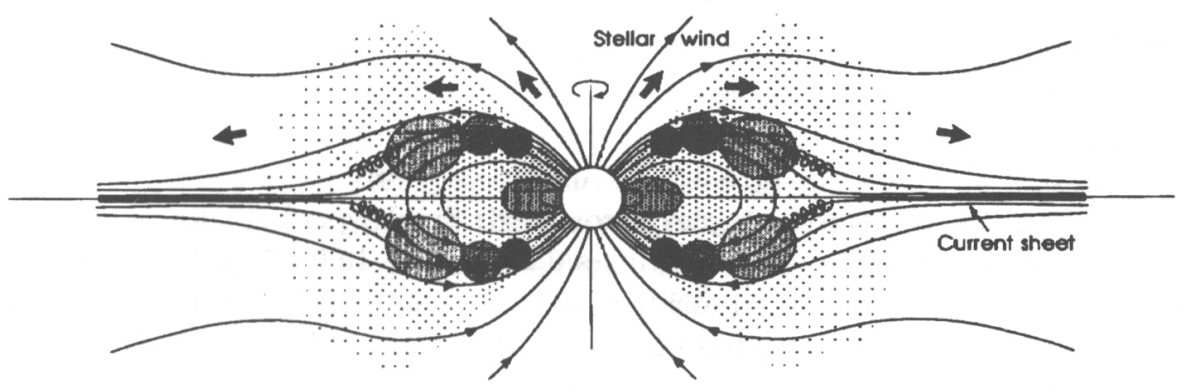

Fig. 1. A cartoon showing a cross section through a CP star and its magnetosphere. For simplicity, an aligned rotator is shown. A dense stipple indicates the presence of dense, trapped thermal plasma. A light stipple indicates the possible presence of tenuous thermal plasma. The small dark circles show schematically where high frequencies originate; larger, lighter circles designate the location of the source with decreasing frequency.

proposed by Morris, Mutel and Su (1990), on the other hand, plasma is trapped in the strong field region of the corona near the star and some of the trapped electrons are accelerated when the field lines are sheared by convective motions at their footpoints. The model proposed by Linsky et al. for the nonthermal radio emission from magnetic CP stars may also have some relevance to certain of the weak T Tauri stars and other PMS objects (e.g., André et al. 1991).

\section{SECOND TEST OF CONVENTIONAL WISDOM: THE SEARCH FOR CORONAL X-RAY EMISSION}

\section{What Has Been Learned From Einstein}

In their initial survey of stellar x-ray emission using the Einstein satellite, Vaiana et al. (1981) first called attention to a significant gap in the X-ray H-R diagram at the early-B stars where no stars were detected as $\mathrm{x}$-ray sources, and explained this gap as being due to the absence of convection in these stars. They did list a few late-B and A-type stars as X-ray detections, however, including both Sirius and Vega. Shortly thereafter Cash and Snow (1982) used Einstein to observe 20 A-type CP stars. Of those stars that were not known to be binaries, only two Ap stars (the $\mathrm{SrCr}$ star $\omega \mathrm{Oph}$ and the $\mathrm{HgMn}$ star $\beta \mathrm{Scl}$ ) were detected with $\mathrm{x}$-ray luminosities of $\log \mathrm{L}_{x}=28.4$ and 28.8 , respectively. Cash and Snow were concerned, however, that the $\mathrm{x}$-ray emission could come from previously unknown cool dwarf companions that are optically faint but $\mathrm{x}$-ray bright (see Table II). 
TABLE II. Comparison of Radio Parameters of Different Types of Stars

\begin{tabular}{cccccc}
\hline Parameter & Sun & dMe & RS CVn & He-S & He-W \\
\hline Spectral type & G2 V & dM4e & K0 IV & B2 V & B8 V \\
$R_{\star} / R_{\odot}$ & 1.0 & 0.4 & 3 & 4 & 3 \\
$\mathrm{~B}_{\text {photo }}(\mathrm{kG})$ & 1.5 & $3-5$ & $\approx 1$ & $2.0-10.0$ & $\leq 1.0-35.0$ \\
$f_{\text {photo }}$ & 0.02 & $0.2-0.7$ & $0.3 ?$ & $\approx 1$ & $\approx 1$ \\
Topology & complex & complex & complex & dipole/quad & dipole \\
$<f B_{\text {photo }}>$ & 0.03 & $0.6-3.5$ & $\approx 0.3$ & $2.0-10.0$ & $\leq 1.0-35.0$ \\
$<f B_{\text {photo }}>R_{\star}^{2}$ & 0.03 & $0.1-0.6$ & $\approx 3$ & $32-160$ & $\leq 9.0-315$ \\
$\log \mathrm{L}_{6}$ & 11.0 & $13.0-14.6$ & $14.5-18.0$ & $17.4-17.9$ & $15.7-17.9$ \\
$\log \mathrm{L}_{x}$ & 27.7 & $27.7-29.8$ & $29.5-32.0$ & 30.2 & 30.0 \\
$\log \mathrm{L}_{x} / \mathrm{L}_{\text {bol }}$ & -6 & -4 to -2 & -6 to -3 & -7 & -6 \\
flaring & yes & yes & yes & no? & no? \\
active regions & yes & yes & yes & - & - \\
$\left(R / R_{\star}\right)^{2} T_{B}$ & $8 \times 10^{4}$ & $1 \times 10^{8}$ & $1 \times 10^{10}$ & $2 \times 10^{10}$ & $0.04-6 \times 10^{10}$ \\
$R / R_{\star}$ & 1 & - & 3 & $<4$ & - \\
$T_{B}$ & $8 \times 10^{4}$ & - & $1 \times 10^{9}$ & $\geq 1 \times 10^{9}$ & - \\
\hline \hline
\end{tabular}

This concern and also the problem of ultraviolet light leaks in the Einstein HRI detector have frustrated attempts to determine whether any $\mathrm{CP}$ or chemically normal A- and B-type main sequence stars are coronal $\mathrm{X}$-ray sources. For example, Golub et al. (1983) found no additional single CP stars as x-ray sources, but identified Sirius A (A1 V), Vega (A0 V), and Altair (A7 IV-V) as $\mathrm{X}$-ray sources. Schmitt et al. (1985) showed that the $\mathrm{x}$-ray detections of the first two stars were actually detections of stellar ultraviolet light by the Einstein HRI, but they argued that the x-ray detection of Altair was not likely to be due to a cooler companion star because the inferred temperature of the emitting plasma was much lower than is invariably found for active late-type stars. In their study of the $\mathrm{x}$-ray emission from late- $\mathrm{A}$ and early $\mathrm{F}$-type field stars, Schmitt et al. (1985) concluded that the detections of a few A-type stars with the Einstein IPC can be explained by x-ray emission from previously unknown $M$ dwarf companions, since the measured $\log \mathrm{L}_{x} \sim 29.0$ is consistent with active M dwarf coronae.

In the young Ursa Major cluster, Schmitt et al. (1990) identified one single A-type star, $\beta$ Eri (A3 III) as an x-ray source, but unlike Altair, its x-ray temperature is similar to that found in a typical F-or G-type giant and thus a companion of this type could be the emission source. All five detected Atype stars in the Hyades Cluster are known to be members of multiple systems (Micela et al. 1988) so that their x-ray properties are in doubt. As one proceeds to younger clusters such as the Pleiades (Micela et al. 1990) and the Orion Nebula (Caillault and Zoonematkermani 1989), the detected A-type stars are far more $\mathrm{x}$-ray luminous than field A-type stars and thus more likely than a putative late-type companion to be the $\mathrm{x}$-ray source. However, $\mathrm{x}$-ray luminous weak-line $\mathrm{T}$ Tauri stars are possible companions for stars as young as those in Orion. 
The early B-type stars present a different situation. Caillault and Zoonematkermani (1989) identified seven early-B stars in the Orion Nebula as luminous $\mathrm{x}$-ray sources, but their $\mathrm{L}_{x} / \mathrm{L}_{\mathrm{bol}}$ ratios lie in the range $10^{-6}-10^{-8}$ that characterizes the 0 -type stars for which the $\mathrm{x}$-ray emission is thought to be formed in shocks in their radiation driven winds rather than in magnetically heated coronae. Grillo et al. (1992) searched the whole Einstein archive to identify 74 B-type $\mathrm{x}$-ray sources. Most of these have spectral types B0-B3 with $\mathrm{L}_{x} / \mathrm{L}_{\mathrm{bol}}$ ratios in the range $10^{-6}-10^{-8}$. These are likely to be stars with $\mathrm{x}$-rays produced in their winds. On the other hand, 34 of 1058 stars in the spectral range B5-B9 were detected as $\mathrm{X}$-ray sources. Grillo et al. conclude that $\mathrm{X}$-ray emission from the late-B stars is much less common than in the hotter stars, but whether the emission comes from late-type companions of these late-B stars is not yet known.

\section{New Information From ROSAT}

The previously described investigations with the Einstein satellite left unanswered the question of whether the CP stars are x-ray sources. Several investigations with the ROSAT satellite are attempting to answer this question. The first of these to be completed is the search for x-ray emission from all $102 \mathrm{CP}$ stars with radio detections or upper limits. Drake et al. (1992) searched the ROSAT all-sky survey, which has a detection threshhold of $3 \times 10^{-13} \mathrm{ergs}^{-2}$ $\mathrm{s}^{-1}$ in about $600 \mathrm{~s}$ integration, detecting $\mathrm{x}$-ray sources at the locations of 11 $\mathrm{CP}$ stars. Four of these stars have hotter companions that are likely wind $\mathrm{x}$-ray sources. Of the remaining seven stars, HR 3089 and $\theta$ Car are early-B He-S stars. The $\mathrm{x}$-ray emission from these two stars is probably produced in their winds as the $\mathrm{L}_{x} / \mathrm{L}_{\mathrm{bol}}$ ratios are near $10^{-7}$ like that of the 0 -type stars. The star $52 \mathrm{Her}(\mathrm{A} 2 \mathrm{Vp} \mathrm{SrCrEu})$ is the least $\mathrm{x}$-ray luminous $\left(\log \mathrm{L}_{x}=28.8\right)$ of the group. Since this value of $L_{x}$ is consistent with that of an active $M$ dwarf star, one cannot rule out a possible companion star as the source of emission.

The remaining four stars in the ROSAT all-sky survey sample are the HeW and Si rich stars: $\nu$ For (B9.5p Si), HR 5624 (Ap Si), HR 5988 (B8p He-W), and HR 6054 (B7 IV He-W). They are characterized by $\log \mathrm{L}_{x} \geq 29.5$, which is more luminous than essentially all single $M$ dwarfs stars, and $\mathrm{L}_{x} / \mathrm{L}_{\mathrm{bol}} \geq 10^{-6.3}$, which makes it unlikely that the $\mathrm{x}$-rays are produced in stellar winds as in the O-type stars. Drake et al. therefore concluded that these four stars are the first detected members of a new class of coronal x-ray CP stars. Two of these stars (HR 5988 and HR 6054) have detected nonthermal radio emission. Their radio and $\mathrm{x}$-ray luminosities are plotted in Fig. 2 along with those of the RS CVn systems for which Dempsey et al. (1992) have obtained ROSAT all-sky survey data and for which radio data are available. The radio fluxes of the other two stars are now being reduced. The two $\mathrm{CP}$ stars with detected radio and $\mathrm{x}$-ray emission have luminosities consistent with the distribution of RS CVn systems (see Fig. 2) but not the $M$ dwarfs, which typically have $\log L_{6} \leq 14.6$ and $\log L_{x} \leq 29.5$. This reinforces the conclusion that the $x$-ray and radio emission 


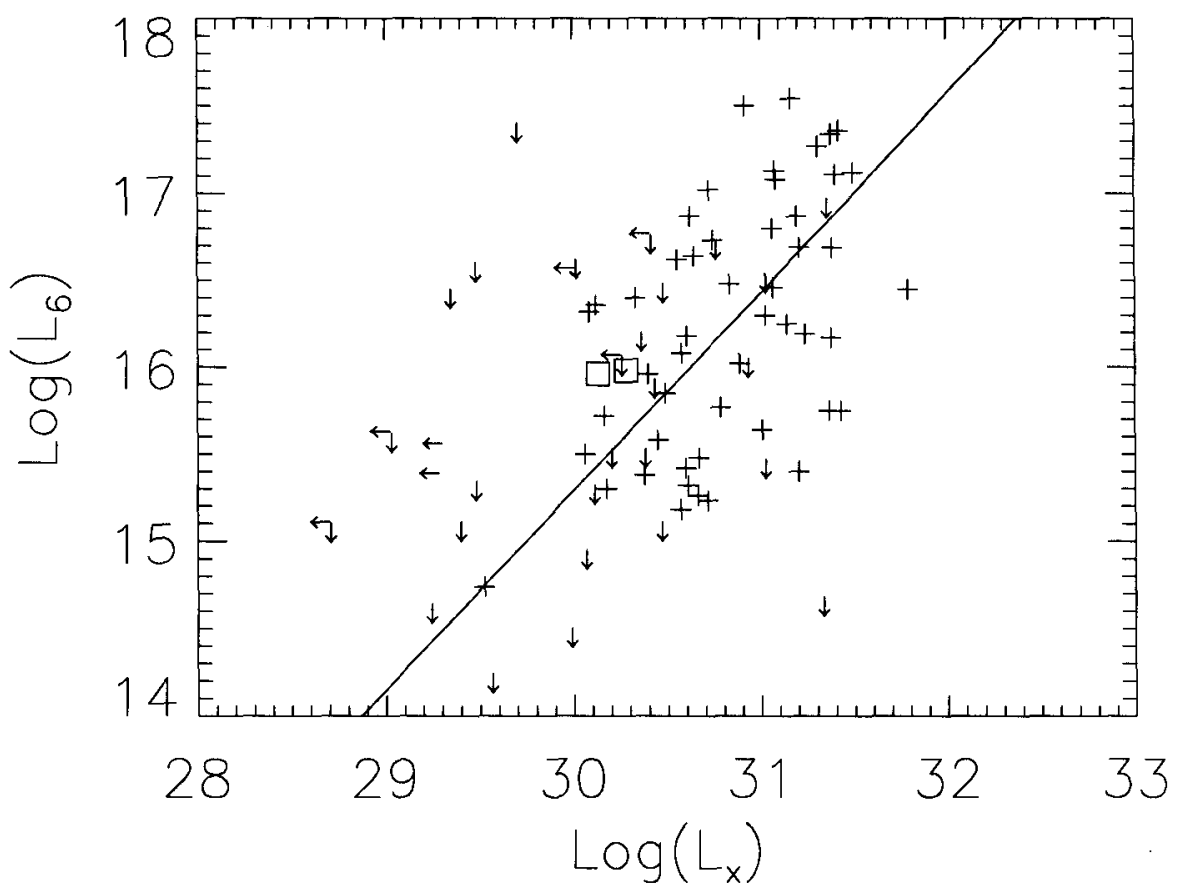

Fig. 2. Comparison of radio and ROSAT $x$-ray emission for CP stars (squares) and RS CVn systems (pluses). Upper limits are indicated by arrows, and the solid line is a maximum likelihood fit to the RS CVn data. The data for the RS CVn systems are from Dempsey et al. (1992).

are intrinsic to the $\mathrm{CP}$ stars rather than to unknown $\mathrm{M}$ dwarf companions, and that the $\mathrm{x}$-ray and radio emission from the $\mathrm{CP}$ stars have similar properties to those of the most active of the late-type stars, the RS CVn systems.

The positive identification of four $\mathrm{He}-\mathrm{W}$ stars as $\mathrm{x}$-ray sources strengthens the hypothesis advanced by Mitskevich and Tsymbal (1992) that the weakness of the helium lines among the late-B CP stars is not due to a true deficiency of helium, but rather to the "superionization" of He I by $\mathrm{x}$-ray and XUV photons shining down on the photosphere from a hot corona. They find that the He I lines are most affected for stars in the effective temperature range $14,000 \mathrm{~K}<$ $\mathrm{T}_{\text {eff }}<20,000 \mathrm{~K}$, which is appropriate for the late-B and middle-B stars, but that the effect becomes unimportant for stars with $\mathrm{T}_{\text {eff }}>25,000 \mathrm{~K}$, which is appropriate for stars near spectral type B0 V. The measurement of $\mathrm{X}$-ray fluxes from a larger sample of CP stars is needed to test this interesting hypothesis. 


\section{CONCLUSIONS}

My story is not complete as pointed x-ray observations are needed to study the $\mathrm{x}$-ray time variability and coronal plasma temperatures of $\mathrm{CP}$ stars, and additional radio observations, including VLBI observations, are needed to better understand the sizes and nonthermal electron energies in the magnetospheres of these stars. Nevertheless, the case is now made that at least some CP stars display phenomena very similar to the most active late-type stars. We have developed a wind-fed magnetosphere model that can account for both the nonthermal radio and coronal $x$-ray emission by using the wind as a mechanical energy source analogous to the convective motions in the late-type stars. This model should be modified to include oblique magnetic geometries and to make quantitative predictions of the $\mathrm{x}$-ray emission. I encourage CP star theorists to accept this challenge.

\section{ACKNOWLEDGMENTS}

I thank NASA for partial support of this work through Interagency Transfers W-17,772 and H-04630D to the N.I.S.T. and grant NAG5-1797 to the University of Colorado. I also thank Dr. Stephen Drake and Robert Dempsey for permission to cite our ROSAT results prior to publication. I also thank Dr. Drake for his comments on the manuscript.

\section{REFERENCES}

André, P., Phillips, R. B., Lestrade, J.-F., and Deeney, B. 1991, this volume.

Babcock, H.W. 1958, ApJS 3, 141.

Caillault, J.-P. and Zoonematkermani, S. 1989, ApJL 338, L57.

Cash, W. and Snow, T.P.Jr. 1982, ApJL 263, L59.

Dempsey, R.C., Linsky, J.L., Schmitt, J.H.M.M., and Fleming, T.A. 1992, submitted to ApJS.

Drake, S.A., Abbott, D.C., Bastian, T.S., Bieging, J.H., Churchwell, E., Dulk, G., and Linsky, J.L. 1987 ApJ 322, 902.

Drake, S.A., Abbott, D.C., Bieging, J.H., Churchwell, E., and Linsky, J.L. 1985, in Radio Stars, ed. R.M. Hjellming and Gibson, D.M. (Dordrecht: D. Reidel), p. 247.

Drake, S.A., Linsky, J.L., Schmitt, I.H.M.M., and Rosso, C. 1992, submitted to ApJL.

Golub, L., Harnden, F.R.Jr., Maxson, C.W., Rosner, R., Vaiana, G.S., Cash, W.Jr., and Snow, T.P.Jr. 1983, ApJ 271, 264.

Grillo, F., Sciortino, S., Micela, G., Vaiana, G.S., and Harnden, F.R.Jr. 1992, ApJS 81, 795.

Havnes, O., and Goertz, C.K. 1984, A\&A 138, 421.

Leone, F. 1991, A\&A. 252, 198. 
Linsky, J.L., Drake, S.A., and Bastian, T.S. 1992, ApJ 393, 341.

Luyten, W.J. 1949, ApJ 109, 532.

Micela, G., Sciortino, S., Vaiana, G.S., Harnden, F.R.Jr., Rosner, R., and Schmitt, J.H.M.M. 1990, ApJ 348, 557.

Micela, G., Sciortino, S., Vaiana, G.S., Schmitt, J.H.M.M., Stern, R.A., Harnden, F.R.Jr., and Rosner, R. 1988, ApJ 325, 798.

Mitskevich, A.S. and Tsymbal, V.V. 1992, A\&A, in press.

Morris, D.H., Mutel, R.L., and Su, B. 1990, ApJ 362, 299.

Schmitt, J.H.M.M., Golub, L., Harnden, F.R.Jr., Maxson, C.W., Rosner, R., and Vaiana, G.S. 1985, ApJ 290, 307.

Schmitt, J.H.M.M., Micela, G., Scortino, S., Vaiana, G.S., Harnden, F.R.Jr., and Rosner, R. 1990, ApJ 351, 492.

Strassmeier, K.G., Hall, D.S., Zeilik, M., Eker, Z., and Fekel, F.C. 1988, A\&AS 72, 291.

Vaiana, G.S. et al. 1981, ApJ 244, 163. 